\title{
A numerical study on MHD double diffusive nonlinear mixed convective nanofluid flow around a vertical wedge with diffusion of liquid hydrogen
}

\author{
Prabhugouda Mallanagouda Patil ${ }^{1,2^{*}}$ and Madhavarao Kulkarni ${ }^{1}$
}

\author{
*Correspondence: \\ pmpmath@gmail.com; \\ pmpatil@kud.ac.in \\ ${ }^{1}$ Department \\ of Mathematics, Karnatak \\ University, Pavate Nagar, \\ Dharwad 580003, India \\ Full list of author information \\ is available at the end of the \\ article
}

\begin{abstract}
The present study focuses on double diffusive nonlinear (quadratic) mixed convective flow of nanoliquid about vertical wedge with nonlinear temperature-density-concentration variations. This study is found to be innovative and comprises the impacts of quadratic mixed convection, magnetohydrodynamics, diffusion of nanoparticles and liquid hydrogen flow around a wedge. Highly coupled nonlinear partial differential equations (NPDEs) and boundary constraints have been used to model the flow problem, which are then transformed into a dimensionless set of equations utilizing non-similar transformations. Further, a set of NPDEs would be linearized with the help of Quasilinearization technique, and then, the linear partial differential equations are transformed into a block tri-diagonal system through using implicit finite difference scheme, which is solved using Verga's algorithm. The study findings were explored through graphs for the fluid velocity, temperature, concentration, nanoparticle volume fraction distributions and its corresponding gradients. One of the important results of this study is that the higher wedge angle values upsurge the friction between the particles of the fluid and the wedge surface. Rising Schmidt number declines the concentration distribution and enhances the magnitude of Sherwood number. Nanofluid's temperature increases with varying applied magnetic field. The present study has notable applications in the designing and manufacturing of wedge-shaped materials in space aircrafts, construction of dams, thermal systems, oil and gas industries, etc.
\end{abstract}

Keywords: Magnetohydrodynamic flow (MHD), Nanoliquid, Quadratic convection, Quasilinearization technique

\section{Introduction}

The linear variations in temperature, density and species concentration relations, i.e., mixed convection flows have been studied by a many number of researchers [1-5]. However, the study of nonlinear changes in density temperature and species concentration relation (NDTC) [6-9] is novel concept for researchers to investigate the effects of the fluid's thermal attributes. The quadratic convection plays an important role in many fields of industries, including thermal systems, combustion, geothermal power plants, author(s) and the source, provide a link to the Creative Commons licence, and indicate if changes were made. The images or other third party material in this article are included in the article's Creative Commons licence, unless indicated otherwise in a credit line to the material. If material is not included in the article's Creative Commons licence and your intended use is not permitted by statutory regulation or exceeds the permitted use, you will need to obtain permission directly from the copyright holder. To view a copy of this licence, visit http:// creativecommons.org/licenses/by/4.0/. 
crude oil extraction, electronic part cooling, pharmaceuticals, and so on. Particularly, the manufacturing and designing of the disk of gate valves, which are effective at high temperature elucidate the phenomenon of nonlinearity in temperature, concentration and density variations. Because of its physical effects on fluid flow and heat transport characteristics, nonlinearity has piqued the interest of many researchers. Sinha [6] has looked at the impact of frictional heating systems and heat sources on convective flows using NDT variations. Vajravelu and Sastri [7] have discussed the energy transmission between surfaces comprising quadratic convection. Kameswaran et al. [8] investigated quadratic convection flow through a non-Darcy permeable surface. Shaw et al. [9] have analyzed quadratic changes in temperature and density relation variations along the stretched surface and reported that the increasing nonlinear convection coefficient values increase the magnitude of transfer of heat from the wall. Hayat et al. [10] have discussed the influence of hydromagnetic on 3-D quadratic convective flow of nanoliquid over a stretched sheet, reported that temperature and heat transport rate increase due to rising values of thermal radiation and temperature ratio parameter. Khan et al. [11] have addressed the quadratic mixed convective of nanoliquid flow with hyperbolic entropy generation on stretched surface and their results unveil that the nonlinear convection parameter rises the magnitude of the velocity pattern. Furthermore, Hayat et al. [12] and Uddin et al. [13] have discussed the major influence of nonlinear convective flow on a horizontally stretched cylinder and surface.

It is worth to note that in the industrial and engineering applications, the flow over a wedge geometry has gained an extensive application in the oil and gas industries, pharmaceuticals, aerospace, construction of dams, designing of disk of engine gate valves, thermal insulation, etc. A milestone contribution of fluid flow along a wedge was given by Falkner and Skan [14]. Kafoussias and Nanousis [15] have examined the hydromagnetic flow along a permeable wedge. Kumari et al. [16] explored combined convective flow through a porous wedge and found that the strength of Nusselt number upsurges with variation in Prandtl number, but the friction coefficient gradually decreases. Kandasamy et al. [17] have explored the transport rate of heat on a porous, as well as permeable wedge, and they reported that the concentration profile declines for increasing chemical reaction parameter values. Singh et al. [18] have considered the time-subordinate combined convective stream over a vertical wedge. Uddin and Kumar [19] have addressed the Hall and ion slip impacts on the MHD flow of micropolar liquid around a wedge and their outcomes reveal that the surface friction increases significantly for higher values of wedge angle. Further, more works on convective boundary layer stream over a wedge can be seen in $[20,21]$.

Owing to breadth of applications in science and technology, a new fluid came into existence and substantially enhances the thermal conductivity of base fluid. This fluid is referred to as "nanofluid" and it contains nano-meter-sized particles that are distributed in conventional heat transfer basic fluids [22]. The heat transfer coefficient between the heat transfer surface and the heat transfer medium is strongly influenced by the thermal conductivity of these typical base fluids. Convective heat transfer in nanofluids has been studied by numerous researchers in various geometries. Recently, Gorla et al. [23] have examined combined convective flow of nanoliquid on a porous wedge, and their outcomes show that the increasing Brownian diffusion increases the 
velocity of the nanofluid significantly. Kahalerras et al. [24] implemented a numerical study of combined convection heat transfer and entropy generation in a vertical channel for $\mathrm{Cu}-\mathrm{H}_{2} \mathrm{O}$ nanofluid. The multidiffusive diffusive convective flow of Maxwell nanofluid between two impermeable plates was investigated by Singh et al. [25]. They also considered the passive control of nanoparticle flux at the boundaries, as well as the concentration flux assumed in Stefan's flow. Rahman et al. [26], Khan et al. [27], and Kasmani et al. [28-30] have also looked into the nanofluid flow around a wedge surface.

Double-diffusive mixed convection arises when the fluid is subjected to two distinct unlike density gradients having different rates of diffusion [31,32]. In this paper, the double diffusion occurs due to the diffusion of heat and liquid gas hydrogen along with the diffusions of temperature and concentration. The influence of magnetohydrodynamics (MHD) is also considered, which controls the surface flow and the thermal transport characteristics of the flow [33-36]. Such hydromagnetic flow and its heat transport characteristics have wide spectrum of uses in the metallurgical processes, metal working processes, electronic devices, cooling of nuclear reactors and other industrial processes, etc. Recently, many researchers have carried out their research on MHD double diffusive convection flow over various geometry with different aspects, in order to analyze the heat transport characteristics of the fluid flow. For example, Rashidi et al. [37], Patil et al. [38, 39], Prasad et al. [40], Quiang et al. [41], Mahajan and Sharma [42] have respectively studied on vertical plate, exponentially stretching surface, inclined plate, infinite vertical parallel plates and planes.

The number of scientific articles in the literature review that discuss the importance of consistent flow around the wedge. However, further analysis in this regard is required to explain the influence of nonlinearity, applied magnetic field, diffusion of liquid hydrogen, Brownian motion and thermophoresis with mixed convection, which is the current study's key motivation. Thus, the determination of the work is to examine the interesting aspects of aforementioned studies. The novelties in this study are as follows:

- Analysis of double diffusive nonlinear combined convection with nanoparticles.

- Buongiorno two-phase nanofluid model is taken into account for modeling.

- Influence of applied magnetic field over a wedge.

- Impacts of diffusion of liquid hydrogen are analyzed.

The outcomes of this study have notable applications in the designing and manufacturing of wedge-shaped materials in space aircrafts, construction of dams, thermal systems, oil and gas industries, etc., where nonlinear mixed convection can assist to improve the heat and mass transfer in manufacturing of wedge-shaped bodies by providing various control parameters. Further, in the manufacturing industries, the nanoparticles and liquid hydrogens are also added to the host fluid to regulate the rate of heat and mass transfer during the structuring and assembling of wedge-shaped materials. In this problem, non-similar transformations are used to solve fluid flow conservation equations. Then, solution of these eqautions has been solved through the technique of Quasilinearization along with the implicit FDM [43, 44]. The achieved 
numerical outcomes are verified with Kumari et al. [16], as well as Singh et al. [18], and the comparison shows the good agreement of present results with previous findings by $[16,18]$.

\section{Methods}

\section{Analysis and formulation}

A steady, laminar incompressible fluid about a vertical wedge is considered in this analysis. The flow is along the wedge in the direction of $x$-axis and $u$ be its corresponding velocity, while, the co-ordinate axis $y$ is normal to it and $v$ be its corresponding velocity component and $\left(\frac{\pi \alpha}{2}\right)$ be the half angle of the wedge. The gravitational force acts vertically in the downward direction. Further, $U_{e}=U_{\infty} \bar{x}^{m}$ indicates the velocity, where exponent $m$ is the wedge angle parameter, $\Omega$ indicates the total angle of the wedge, $\alpha$ indicates the Hartree pressure gradient. Further, relation between wedge angle parameter and pressure gradient is given as $m=\frac{\alpha}{2-\alpha} \geq 0$ [27-30]. Here, $T_{w}, C_{w}, \phi_{w}$, respectively, denote temperature, concentration and nanoparticles volume fraction at the surface, while $T_{\infty}$ $, C_{\infty}, \phi_{\infty}$, respectively, denote the same at mainstream region. The flow model of present problem with the coordinate system is as displayed in Fig. 1.

For the more comprehensive study of the mathematical model, the following main features are employed:

- The magnetic field $B_{0}$ is taken perpendicular to a wedge surface.

- The Boussinesq approximation [45] is used to model the nonlinear density temperature concentration terms, which are embedded in the momentum equation.

- In absence of turbulent flow, the present problem is considered with thermophoresis and Brownian diffusion under the slip mechanism of Buongiorno two-phase model [46].

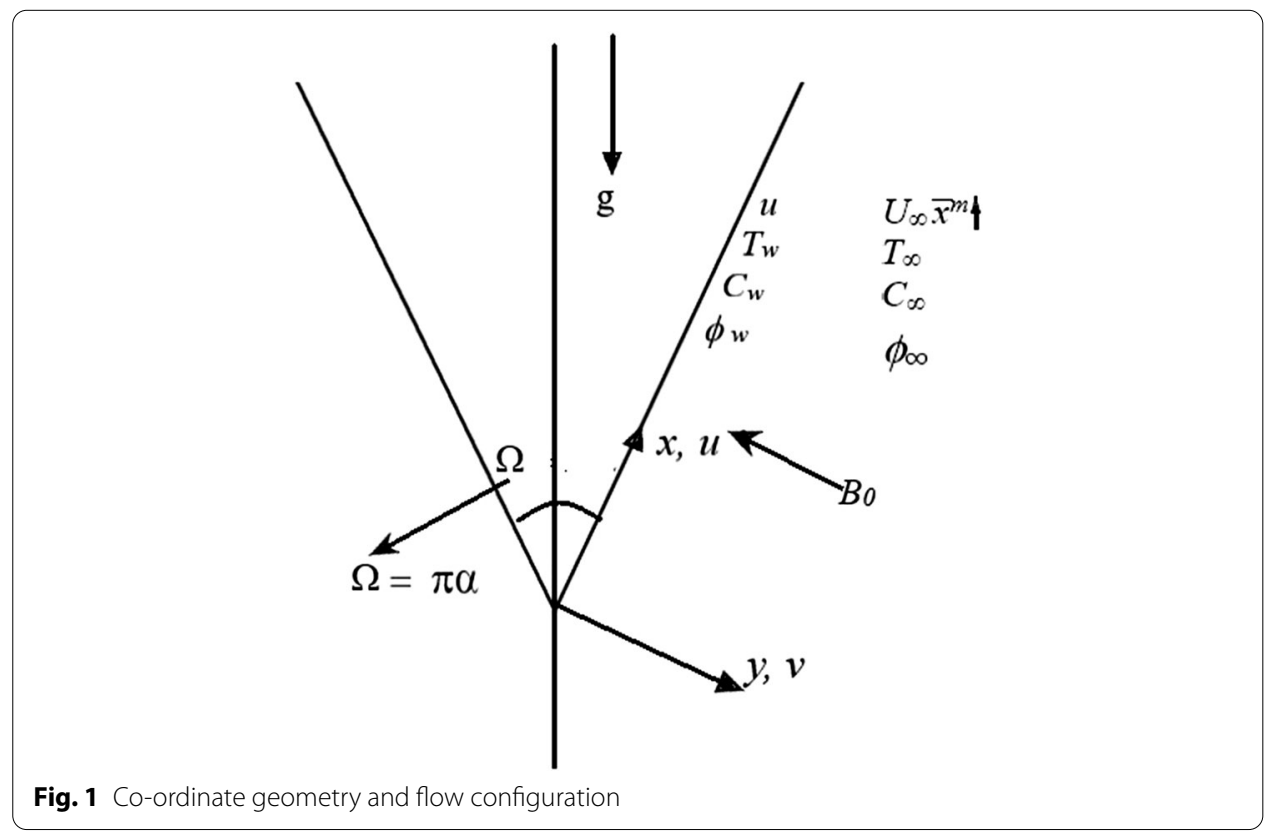


The flow problem is modeled through conservation laws and the governing equations under steady-state conditions are $[6-8,16-18]$ :

$$
\begin{aligned}
& \frac{\partial u}{\partial x}+\frac{\partial v}{\partial y}=0 \\
& u \frac{\partial u}{\partial x}+v \frac{\partial u}{\partial y}=v \frac{\partial^{2} u}{\partial y^{2}}+U_{e} \frac{\mathrm{d} U_{e}}{\mathrm{~d} x}+\left(1-\phi_{\infty}\right) g\left[\beta_{1}\left(T-T_{\infty}\right)+\beta_{2}\left(T-T_{\infty}\right)^{2}\right] \cos \left(\frac{\pi \alpha}{2}\right) \\
& +\left(1-\phi_{\infty}\right) g\left[\beta_{3}\left(C-C_{\infty}\right)+\beta_{4}\left(C-C_{\infty}\right)^{2}\right] \cos \left(\frac{\pi \alpha}{2}\right)+\frac{\sigma B_{0}^{2}}{\rho}\left(U_{e}-u\right) \\
& -g\left(\frac{\rho_{p}-\rho}{\rho}\right)\left(\phi-\phi_{\infty}\right) \cos \left(\frac{\pi \alpha}{2}\right) \\
& u \frac{\partial T}{\partial x}+v \frac{\partial T}{\partial y}=\alpha_{m} \frac{\partial^{2} T}{\partial y^{2}}+\frac{\sigma B_{0}^{2}}{\rho C_{n f}}\left(U_{e}-u\right)^{2}+\frac{\rho_{p} C_{p p}}{\rho C_{n f}}\left(\frac{D_{T}}{T_{\infty}}\left(\frac{\partial T}{\partial y}\right)^{2}+D_{B} \frac{\partial \phi}{\partial y} \frac{\partial T}{\partial y}\right), \\
& u \frac{\partial C}{\partial x}+v \frac{\partial C}{\partial y}=D_{s}\left(\frac{\partial^{2} C}{\partial y^{2}}\right) \\
& u \frac{\partial \phi}{\partial x}+v \frac{\partial \phi}{\partial y}=D_{B}\left(\frac{\partial^{2} \phi}{\partial y^{2}}\right)+\frac{D_{T}}{T_{\infty}}\left(\frac{\partial^{2} T}{\partial y^{2}}\right) .
\end{aligned}
$$

The pertinent surface conditions are as follows:

$$
\begin{aligned}
& y=0: \quad u=0, \quad v=0, \quad T=T_{w}, \quad C=C_{w}, \quad \phi=\phi_{w}, \\
& y \rightarrow \infty: \quad u \rightarrow U_{e}(x)=U_{\infty} \bar{x}^{m}, \quad T \rightarrow T_{\infty}, \quad C \rightarrow C_{\infty}, \quad \phi \rightarrow \phi_{\infty},
\end{aligned}
$$

Utilizing the below non-similar transformations [16-18] in Eqs. (1)-(5):

$$
\begin{aligned}
& \bar{x}=\frac{x}{L}, \quad \eta=\sqrt{\left(\frac{U_{e}}{v x}\right)\left(\frac{m+1}{2}\right)} y, \quad f_{\eta}=F, \\
& T-T_{\infty}=\left(T_{w}-T_{\infty}\right) G(\bar{x}, \eta), \quad C-C_{\infty}=\left(C_{w}-C_{\infty}\right) H(\bar{x}, \eta), \\
& \phi-\phi_{\infty}=\left(\phi_{w}-\phi_{\infty}\right) S(\bar{x}, \eta), \psi(x, y)=\sqrt{\left(\frac{2}{m+1}\right)\left(\nu U_{e} x\right) f(\bar{x}, \eta),} \\
& u=\frac{\partial \psi}{\partial y}, \quad v=-\frac{\partial \psi}{\partial x} .
\end{aligned}
$$

Eq. (1) is satisfied trivially and Eqs. (2)-(5) reduce as below:

$$
\begin{aligned}
& F_{\eta \eta}+f F_{\eta}+\frac{2 \bar{x}}{m+1}\left(f_{\bar{x}} F_{\eta}-F F_{\bar{x}}\right)+\left(\frac{2 m}{1+m}\right)\left(1-F^{2}\right)+\left(\frac{2}{1+m}\right) M^{2} \operatorname{Re}(1-F) N_{2} \\
& +\left(\frac{2 \operatorname{Ri} N_{1}}{1+m}\right)\left[G(1+\gamma G)+\operatorname{Nc} H\left(1+\beta_{c}\right)-\mathrm{Nr} S\right]=0, \\
& G_{\eta \eta}+\operatorname{Pr}\left\{f+\mathrm{Nb} S_{\eta}+\operatorname{Nt} G_{\eta}\right\} G_{\eta}+\frac{2 \operatorname{Pr} \bar{x}}{m+1}\left(f_{\bar{x}} G_{\eta}-F G_{\bar{x}}\right)+\left(\frac{2}{1+m}\right) \operatorname{Pr} M^{2} \operatorname{Ec} \operatorname{Re}(1-F)^{2} N_{3}=0,
\end{aligned}
$$




$$
\begin{aligned}
& H_{\eta \eta}+\operatorname{Sc} f S_{\eta}+\frac{2 \operatorname{Sc} \bar{x}}{m+1}\left(f_{\bar{x}} H_{\eta}-F H_{\bar{x}}\right)=0, \\
& S_{\eta \eta}+\operatorname{Le} f S_{\eta}+\frac{2 \operatorname{Le} \bar{x}}{m+1}\left(f_{\bar{x}} S_{\eta}-F S_{\bar{x}}\right)+\frac{\mathrm{Nt}}{\mathrm{Nb}} G_{\eta \eta}=0 .
\end{aligned}
$$

With help of non-dimensional distance $\xi=(\bar{x})^{\frac{(1-m)}{2}}$ [16-18], Eqs. (8)-(11) reduce as follows:

$$
\begin{aligned}
& F_{\eta \eta}+f F_{\eta}+\left(\frac{1-m}{1+m}\right) \xi\left(f_{\xi} F_{\eta}-F F_{\xi}\right)+\left(\frac{2 \operatorname{Ri} N_{4}}{1+m}\right)\left[G(1+\gamma G)+\mathrm{Nc} H\left(1+\beta_{c}\right)-\mathrm{Nr} S\right] \\
& +\left(\frac{2 m}{1+m}\right)\left(1-F^{2}\right)+\left(\frac{2}{1+m}\right) M^{2} \operatorname{Re}(1-F) N_{5}=0 \\
& G_{\eta \eta}+\operatorname{Pr}\left\{f+\operatorname{Nb} S_{\eta}+\operatorname{Nt} G_{\eta}\right\} G_{\eta}+\operatorname{Pr}\left(\frac{1-m}{1+m}\right) \xi\left(f_{\xi} G_{\eta}-F G_{\xi}\right) \\
& \quad+\left(\frac{2}{1+m}\right) \operatorname{Pr} M^{2} \operatorname{Ec} \operatorname{Re}(1-F)^{2} N_{6}=0 \\
& H_{\eta \eta}+\operatorname{Sc} f H_{\eta}+\operatorname{Sc}\left(\frac{1-m}{1+m}\right) \xi\left(f_{\xi} H_{\eta}-F H_{\xi}\right)=0 \\
& S_{\eta \eta}+\operatorname{Le} f S_{\eta}+\operatorname{Le}\left(\frac{1-m}{1+m}\right) \xi\left(f_{\xi} S_{\eta}-F S_{\xi}\right)+\frac{\mathrm{Nt}}{\mathrm{Nb}} G_{\eta \eta}=0 .
\end{aligned}
$$

where $N_{1}=\bar{x}\left(\frac{U_{\infty}}{U_{e}}\right)^{2}, \quad N_{2}=\bar{x}\left(\frac{U_{\infty}}{U_{e}}\right), \quad N_{3}=\bar{x}\left(\frac{U_{e}}{U_{\infty}}\right), N_{4}=\xi^{\left(\frac{2(1-2 m)}{1-m}\right)}, \quad N_{5}=\xi^{2}, \quad N_{6}=\xi^{\left(\frac{2(1+m)}{1-m}\right)}$.

The surface constraints are as follows:

$$
\begin{aligned}
& F=0, \quad G=1, \quad H=1 \quad S=1, \quad \text { at } \eta=0, \\
& F \rightarrow 1, \quad G \rightarrow 0, \quad H \rightarrow 0, \quad S \rightarrow 0, \quad \text { as } \eta \rightarrow \infty .
\end{aligned}
$$

In nomenclature section, all variables and dimensionless parameters are defined.

Furthermore, $f(\xi, \eta)=\int_{0}^{\eta} F d \eta+f_{w}$, where $f_{w}=0$ represents the impermeable surface. The remaining parameters in this problem are given in nomenclature section.

The gradients at the wedge surface can be expressed as given below:

Local skin friction coefficient : $\operatorname{Re}_{x}^{1 / 2} C_{f}=\sqrt{2(1+m)} F_{\eta}(\xi, 0)$

Local heat transfer rate $: \operatorname{Re}_{x}^{-1 / 2} \mathrm{Nu}=-\sqrt{\frac{(1+m)}{2}} G_{\eta}(\xi, 0)$

Local mass transfer rate $: \operatorname{Re}_{x}^{-1 / 2} S h=-\sqrt{\frac{(1+m)}{2}} H_{\eta}(\xi, 0)$ 
Local nanoparticle mass transfer rate $: \operatorname{Re}_{x}^{-1 / 2} \mathrm{NSh}=-\sqrt{\frac{(1+m)}{2}} S_{\eta}(\xi, 0)$

Further, Eqs. (12)-(15), as well as the boundary constraints (16), are linearized by Quasilinearization technique, yielding the subsequent set of equations:

$$
\begin{aligned}
& F_{\eta \eta}^{i+1}+A_{1}^{i} F_{\eta}^{i+1}+A_{2}^{i} F^{i+1}+A_{3}^{i} F_{\xi}^{i+1}+A_{4}^{i} G^{i+1}+A_{5}^{i} H^{i+1}+A_{6}^{i} S^{i+1}=A_{7}^{i}, \\
& G_{\eta \eta}^{i+1}+B_{1}^{i} G_{\eta}^{i+1}+B_{2}^{i} G_{\xi}^{i+1}+B_{3}^{i} S_{\eta}^{i+1}+B_{4}^{i} F^{i+1}=B_{5}^{i}, \\
& H_{\eta \eta}^{i+1}+C_{1}^{i} H_{\eta}^{i+1}+C_{2}^{i} H_{\xi}^{i+1}+C_{3}^{i} F^{i+1}=C_{4}^{i}, \\
& S_{\eta \eta}^{i+1}+D_{1}^{i} S_{\eta}^{i+1}+D_{2}^{i} S_{\xi}^{i+1}+D_{3}^{i} G_{\eta \eta}^{i+1}+D_{4}^{i} F^{i+1}=D_{5}^{i},
\end{aligned}
$$

where coefficients at the $(i+1)$ th iteration are found through known $i$ th iterative index.

The pertinent surface constraints are as follows:

$$
\begin{aligned}
& F^{i+1}=0, \quad G^{i+1}=1, \quad H^{i+1}=1, \quad S^{i+1}=1, \quad \text { at } \eta=0, \\
& F^{i+1}=1, \quad G^{i+1}=0, \quad H^{i+1}=0, \quad S^{i+1}=0, \quad \text { at } \eta=\eta_{\infty} .
\end{aligned}
$$

Coefficients in Eqs. (21)-(24) are as follows: $A_{1}^{i}=f+\left(\frac{1-m}{1+m}\right) \xi f_{\xi}, A_{1}^{i}=f+\left(\frac{1-m}{1+m}\right) \xi f_{\xi}$, $A_{2}^{i}=-\left\{\left(\frac{1-m}{1+m}\right) \xi F_{\xi}+\frac{4 m}{(1+m)} F+\frac{2 N_{5}}{(1+m)} \operatorname{Re} M^{2}\right\}, \quad A_{3}^{i}=-\left(\frac{1-m}{1+m}\right) \xi F$, $A_{4}^{i}=\left(\frac{2 \operatorname{Ri} N_{4}}{1+m}\right)(1+2 \gamma G), \quad A_{5}^{i}=\left(\frac{2 \operatorname{Ri} N_{4}}{1+m}\right) \mathrm{Nc}\left(1+2 \beta_{c} H\right), \quad A_{6}^{i}=-\frac{2 \operatorname{Ri} N_{4} \mathrm{Nr}}{1+m}$, $A_{7}^{i}=-\left(\frac{2 m}{1+m}\right)\left(1+F^{2}\right)-\left(\frac{1-m}{1+m}\right) \xi F_{\xi} F-\left(\frac{2 N_{5}}{1+m}\right) \operatorname{Re} M^{2}+\left(\frac{2 \operatorname{RiN} N_{4}}{1+m}\right)\left(\gamma G^{2}+\mathrm{Nc} \beta_{c} H^{2}\right)$, $B_{1}^{i}=\operatorname{Pr}\left\{f+\mathrm{Nb} S_{\eta}+2 \mathrm{Nt} G_{\eta}\right\}+\left(\frac{1-m}{1+m}\right) \xi \operatorname{Pr} f_{\xi}, \quad B_{2}^{i}=-\left(\frac{1-m}{1+m}\right) \xi \operatorname{Pr} F, \quad B_{3}^{i}=\operatorname{Pr} \mathrm{Nb} G_{\eta}$, $B_{4}^{i}=-\left(\frac{1-m}{1+m}\right) \xi \operatorname{Pr} G_{\xi}-\left(\frac{4 N_{6}}{1+m}\right) \operatorname{Pr} M^{2} \operatorname{Re} \operatorname{Ec}(1-F)$,

$B_{5}^{i}=\operatorname{PrNt} G_{\eta}^{2}+\operatorname{PrNb} S_{\eta} G_{\eta}-\left(\frac{1-m}{1+m}\right) \xi \operatorname{PrFG} \xi-\left(\frac{2 N_{6}}{1+m}\right) \operatorname{Pr} M^{2} \operatorname{Re} \mathrm{Ec}\left(1-F^{2}\right)$,

$C_{1}^{i}=\operatorname{Sc} f+\left(\frac{1-m}{1+m}\right) \xi \operatorname{Sc} f_{\xi}, \quad C_{2}^{i}=-\left(\frac{1-m}{1+m}\right) \xi \operatorname{Sc} F, \quad C_{3}^{i}=-\left(\frac{1-m}{1+m}\right) \xi \operatorname{Sc} H_{\xi}$, $C_{4}^{i}=-\left(\frac{1-m}{1+m}\right) \xi \operatorname{Sc} F H_{\xi}, \quad D_{1}^{i}=\operatorname{Le} f+\left(\frac{1-m}{1+m}\right) \xi \operatorname{Le} f_{\xi}, \quad D_{2}^{i}=-\left(\frac{1-m}{1+m}\right) \xi \operatorname{Le} F, \quad D_{3}^{i}=\frac{\mathrm{Nt}}{\mathrm{Nb}}$, $D_{4}^{i}=-\left(\frac{1-m}{1+m}\right) \xi \operatorname{Le} S_{\xi}, D_{5}^{i}=-\left(\frac{1-m}{1+m}\right) \xi \operatorname{Le} F S_{\xi}$.

In the implicit finite difference method, for the discretization of Eqs. (21)-(25), the backward and central difference formulas are utilized in $\xi$ and $\eta$ directions. Each iteration step in the block tri-diagonal structure yields the corresponding linear system of equations. These algebraic systems would be solved by the Gauss elimination method, which is not only efficient, it is very stable relative to the growth of rounding errors. Often, an algorithm is referred to as Varga's algorithm [47] to solve these systems of equations and find the inverse of the matrix to get the desired solution of the matrix. Further, this algorithm is restricted only to simple problems and not to high-end simulation problems. Further, numerical solutions' convergence is ensured by optimizing the step length sizes $\Delta \eta$ and $\Delta \xi$. Moreover, the solution is said to have converged when the relative difference between the current and previous iteration values is less than 0.0001 and the iteration process is terminated. 
i.e.,

$\max$ $10^{-4}$

$\left\{\left|\left(F_{\eta}\right)_{w}^{i+1}-\left(F_{\eta}\right)_{w}^{i}\right|,\left|\left(G_{\eta}\right)_{w}^{i+1}-\left(G_{\eta}\right)_{w}^{i}\right|,\left|\left(H_{\eta}\right)_{w}^{i+1}-\left(H_{\eta}\right)_{w}^{i}\right|\left|\left(S_{\eta}\right)_{w}^{i+1}-\left(S_{\eta}\right)_{w}^{i}\right|\right\}<$.

\section{Results and discussion}

In the present analysis, the water is considered as the working fluid, since the Prandtl number Pr value is taken as 7.0. The wedge angle $m=0\left(0^{\circ}\right)$ indicates the flow through a flat plate, whereas $m=0.3333\left(90^{\circ}\right)$ indicates the flow through a vertical plate. As a result, the wedge study is more significant in the range $0<m<0.5$ and the value of $m=0.0909\left(30^{\circ}\right)$ is set in this analysis [16-18]. The liquid gas, namely liquid hydrogen, is regarded as a species concentration, and hence Schmidt numbers, Sc $=160$, are used. The edge of the boundary layer thickness $\left(\eta_{\infty}\right)$ has been taken up to 4.0. Several values have been computed by means of considering $\operatorname{Nr}(0 \leq \mathrm{Nr} \leq 1), M(0 \leq M \leq 1), \xi(0 \leq \xi \leq 1)$, $\gamma(0 \leq \gamma \leq 1), \beta_{c}\left(0 \leq \beta_{c} \leq 1\right), \mathrm{Nt}(0 \leq \mathrm{Nt} \leq 1), \mathrm{Ec}(-0.1 \leq \mathrm{Ec} \leq 0.1), \operatorname{Re}(0 \leq \operatorname{Re} \leq 10)$, $\mathrm{Le}(2 \leq \mathrm{Le} \leq 10), \mathrm{Nc}(0 \leq \mathrm{Nc} \leq 1), \operatorname{Ri}(-1 \leq \mathrm{Ri} \leq 10)$ and $\mathrm{Nb}(0 \leq \mathrm{Nb} \leq 1)$.

\section{Effect of wedge angle parameter}

Figures 2 and 3, respectively, illustrate the variations in velocity distribution $F(\xi, \eta)$ and drag coefficient $\left(\operatorname{Re}_{x}^{1 / 2} C_{f}\right)$ for varying wedge angle parameter $m$ and the Brownian diffusion parameter $\mathrm{Nb}$. It is clear from these graphs augmenting values of $m$ and $N b$ enhance the magnitude of both $F(\xi, \eta)$ and $\left(\operatorname{Re}_{x}^{1 / 2} C_{f}\right)$. Also, the results show the velocity characteristics are overshoot for greater $m$ and $\mathrm{Nb}$ values. As the wedge angle increases, the fluid particles motion increases gradually near the wedge surface. This helps to accelerate the velocity of the nanoliquid and also the magnitude of $\left(\operatorname{Re}_{x}^{1 / 2} C_{f}\right)$. On the other hand, the distributed nanoparticles in the base liquid move randomly and their collision to each other yields faster movement in fluid particles toward the mainstream region. This triggers the rise in the nanoliquid velocity, as well as the drag between the fluid and the wedge's surface.

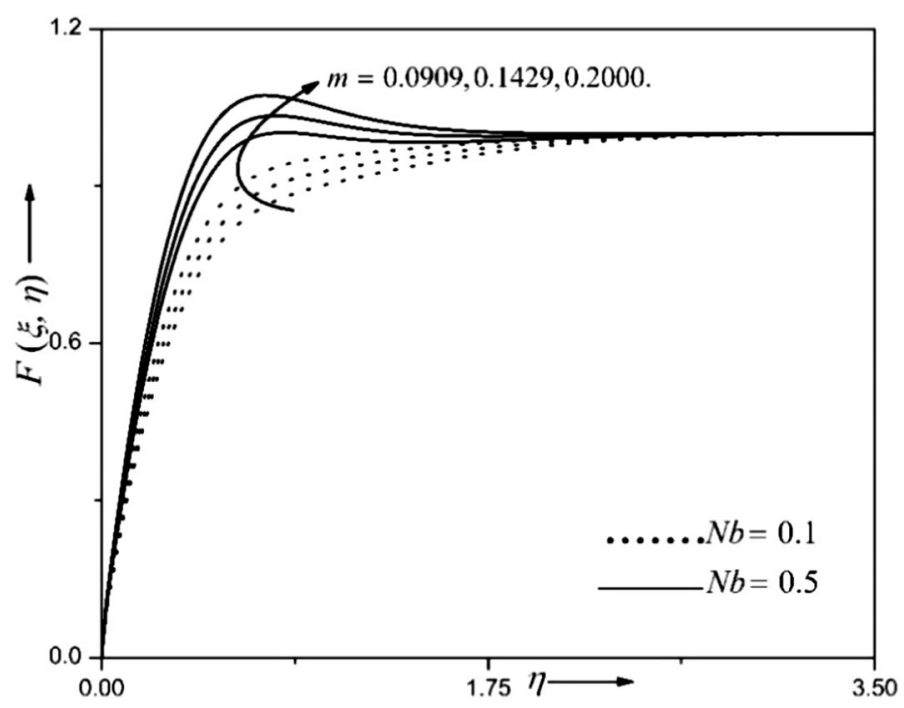

Fig. 2 Influence of $m$ and $\mathrm{Nb}$ on $F(\xi, \eta)$ when $\gamma=0.1, \operatorname{Re}=10, \mathrm{Ri}=10, \xi=0.5, \mathrm{Nt}=0.1, \beta_{c}=0.1$, Le $=10$, $M=0.1, \mathrm{EC}=0.1, \mathrm{NC}=0.1, \mathrm{Pr}=7, \mathrm{SC}=160$ and $\mathrm{Nr}=0.1$ 


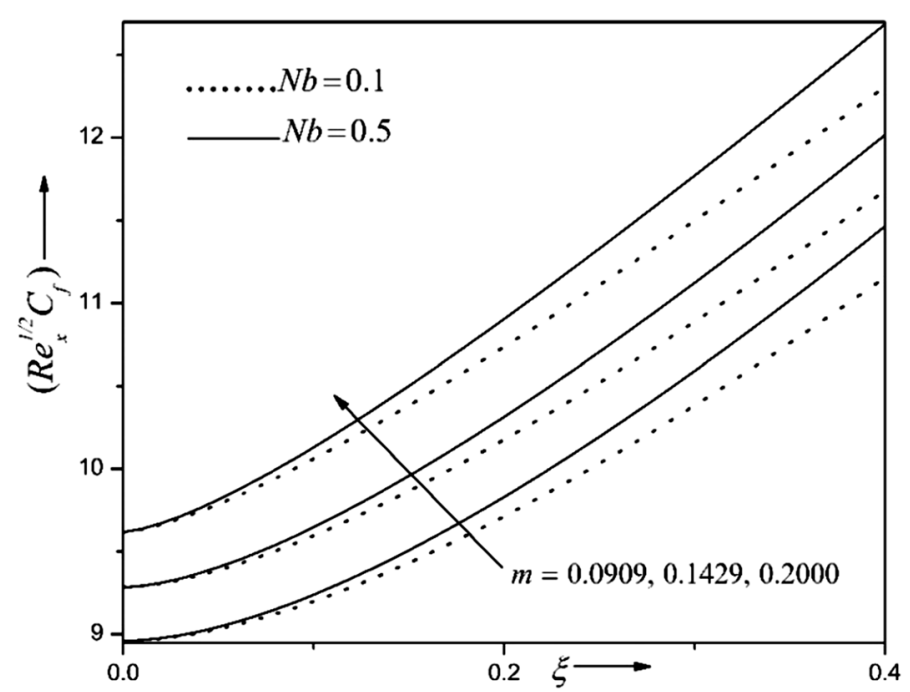

Fig. 3 Influence of $m$ and $\mathrm{Nb}$ on $\left(\operatorname{Re}_{x}^{1 / 2} C_{f}\right)$ when $\mathrm{Le}=10, \mathrm{Nc}=0.1, M=0.1, \mathrm{EC}=0.1, \mathrm{Nt}=0.1, \mathrm{Pr}=7, \mathrm{Sc}=160$, $\gamma=0.1, \mathrm{Ri}=10, \mathrm{Re}=10, \beta_{c}=0.1$ and $\mathrm{Nr}=0.1$

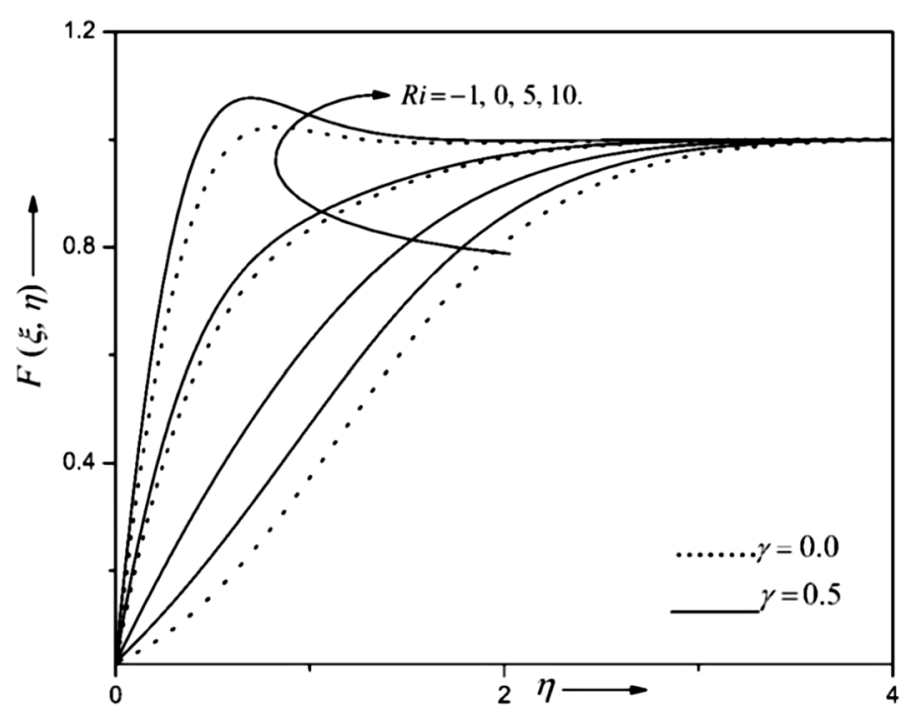

Fig. 4 Influence of $\gamma$ and $\operatorname{Ri}$ on $F(\xi, \eta)$ when $L e=10, N c=0.1, \beta_{c}=0.1, m=0.0909, \operatorname{Re}=10, \mathrm{Nb}=0.1$, $M=0.1, \mathrm{EC}=0.1, \mathrm{Nt}=0.1, \mathrm{SC}=160, \xi=0.5, \operatorname{Pr}=7$ and $\mathrm{Nr}=0.1$

\section{Influence of quadratic mixed convection parameter}

Figures 4 and 5 , respectively, portray the variations in $F(\xi, \eta)$ and $\left(\operatorname{Re}_{x}^{1 / 2} C_{f}\right)$ for varying quadratic combined convection parameter $\gamma$ and Richardson number Ri. The enhancing values of $\gamma$ boost the strength of velocity distribution and coefficient of skin friction. The explanation for this behavior is that growing $\gamma$ values raise the fluid's temperature, which accelerates the fluid particles near the wedge surface and induces such discrepancies in $F(\xi, \eta)$ and $\left(\operatorname{Re}_{x}^{1 / 2} C_{f}\right)$. The reason for this behavior is that the rising values of $\gamma$ rise the fluid's temperature, which accelerates the fluid particles near the wedge surface and causes such variations. In addition to this, the magnitude of velocity profile and 


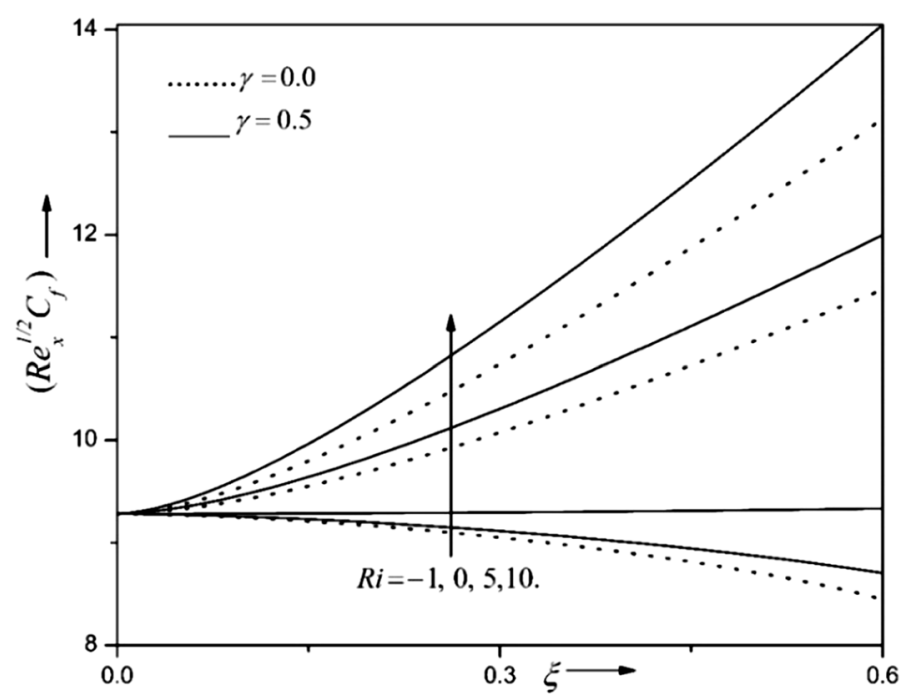

Fig. 5 Influence of $\gamma$ and $\operatorname{Ri}$ on $\left(\operatorname{Re}_{x}^{1 / 2} C_{f}\right)$ for $\mathrm{Nb}=0.1, m=0.0909, \operatorname{Re}=10, \mathrm{SC}=160$, Le $=10, \operatorname{Pr}=7, M=0.1$, $\mathrm{EC}=0.1, \mathrm{Nt}=0.1, \mathrm{Nc}=0.1, \beta_{\mathrm{c}}=0.1$ and $\mathrm{Nr}=0.1$

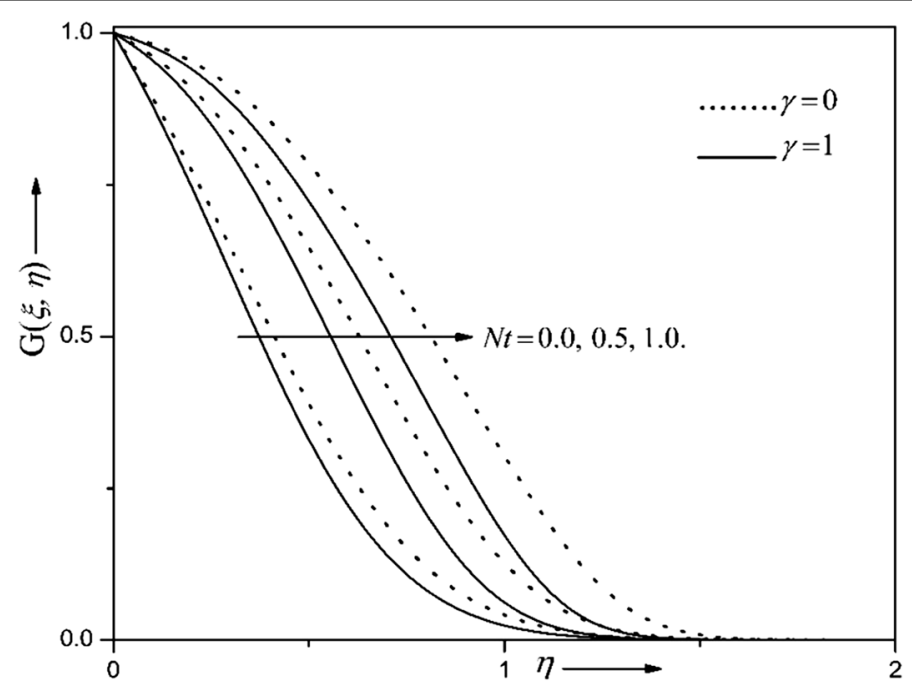

Fig. 6 Variations in $\gamma$ and $\mathrm{Nt}$ on $\mathrm{G}(\xi, \eta)$ when $\mathrm{EC}=0.1, \mathrm{Ri}=10, \xi=0.5, \mathrm{Le}=10, \operatorname{Pr}=7, \beta_{c}=0.1, \mathrm{Nb}=0.1$, $m=0.0909, \operatorname{Re}=10, M=0.1, \mathrm{Nc}=0.1, \mathrm{Sc}=160$ and $\mathrm{Nr}=0.1$

corresponding gradient increase for positive values of Ri and the reverse pattern is perceived for negative Ri. Also, the aiding buoyancy force acts as a favorable pressure gradient, which helps to increase nanoliquid's velocity, as well as friction at the fluid and the wedge surface, while the opposing buoyancy flow acts as an negative pressure gradient and diminishes the $F(\xi, \eta)$, as well as $\left(\operatorname{Re}_{x}^{1 / 2} C_{f}\right)$.

Figures 6 and 7 demonstrate the behavior of quadratic combined convection parameter $\gamma$ and thermophoresis parameter Nt on temperature distribution $G(\xi, \eta)$ and heat transfer rate. According to these graphs, growing nonlinear combined convection parameter values reduce the thermal boundary thickness, which lowers temperature 


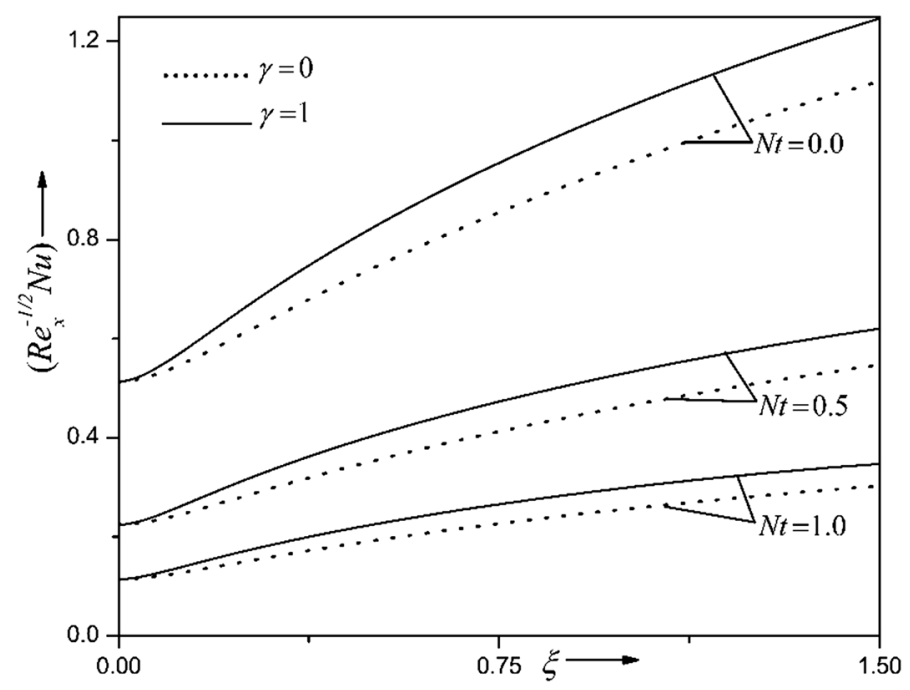

Fig. 7 Influence of $\gamma$ and $\mathrm{Nt}$ on $\left(\operatorname{Re}_{x}^{-1 / 2} \mathrm{Nu}\right)$ when $\operatorname{Re}=10, \mathrm{Le}=10, \mathrm{M}=0.1, \operatorname{Pr}=7, \mathrm{Ri}=10, \mathrm{Nb}=0.1, \mathrm{Sc}=160$, $\mathrm{EC}=0.1, m=0.0909, \mathrm{Nc}=0.1, \beta_{c}=0.1$ and $\mathrm{Nr}=0.1$

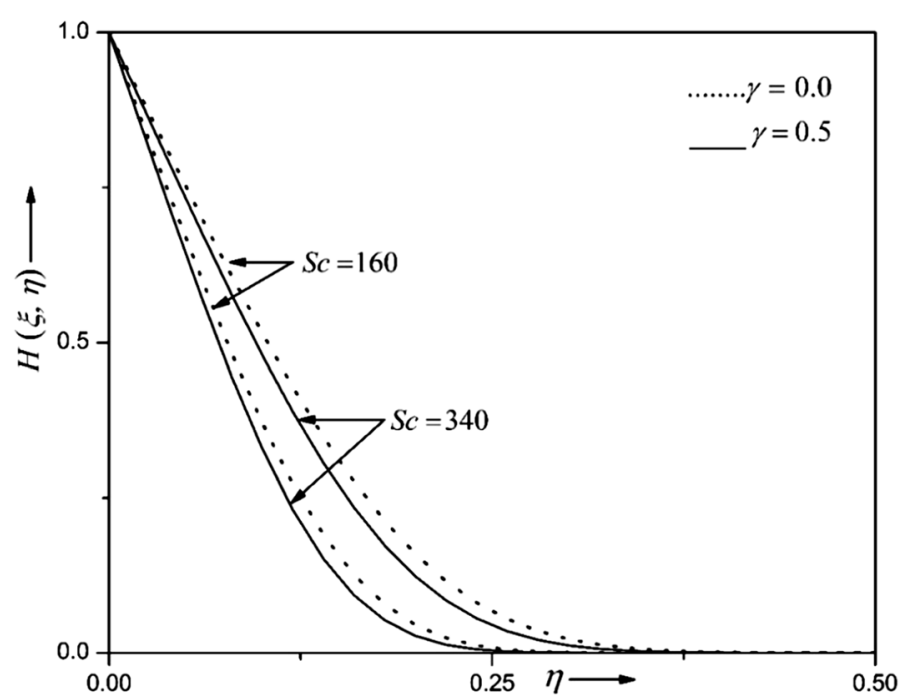

Fig. 8 Effect of $\gamma$ and $\mathrm{Sc}$ on $H(\xi, \eta)$ when $\mathrm{Nb}=0.1, m=0.0909, \mathrm{Re}=10, M=0.1, \mathrm{Ec}=0.1, \mathrm{Ri}=10, \mathrm{Nc}=0.1, \mathrm{Sc}$ $\mathrm{Nt}=0.1, \operatorname{Pr}=7, \xi=0.5$, Le $=10, \beta_{c}=0.1$ and $\mathrm{Nr}=0.1$

of the nanoliquid and thus increases the rate of heat transportation. The difference in temperature $\left(T_{w}-T_{\infty}\right)$ is the reason for such disparities in profile and also in gradient. Moreover, increasing pattern is seen in the dimensionless temperature profile by varying Nt. Thermophoresis is the migration of nanoparticles from hot region to cold one due to temperature gradient, which causes the raise in temperature of the nanofluid. Consequently, transfer of heat around the wedge surface decreases. For example, at $\xi=1$, as Nt upsurges from 0 to 1, the rate of heat transport growths about $70-74 \%$ for $\gamma=1$ and $\gamma=0$, respectively.

Figures 8 and 9 display the variations in concentration profile $H(\xi, \eta)$ and mass transfer $\left(\operatorname{Re}_{x}^{-1 / 2} \mathrm{Sh}\right)$ for varying values of quadratic mixed convection parameter $\gamma$ and 


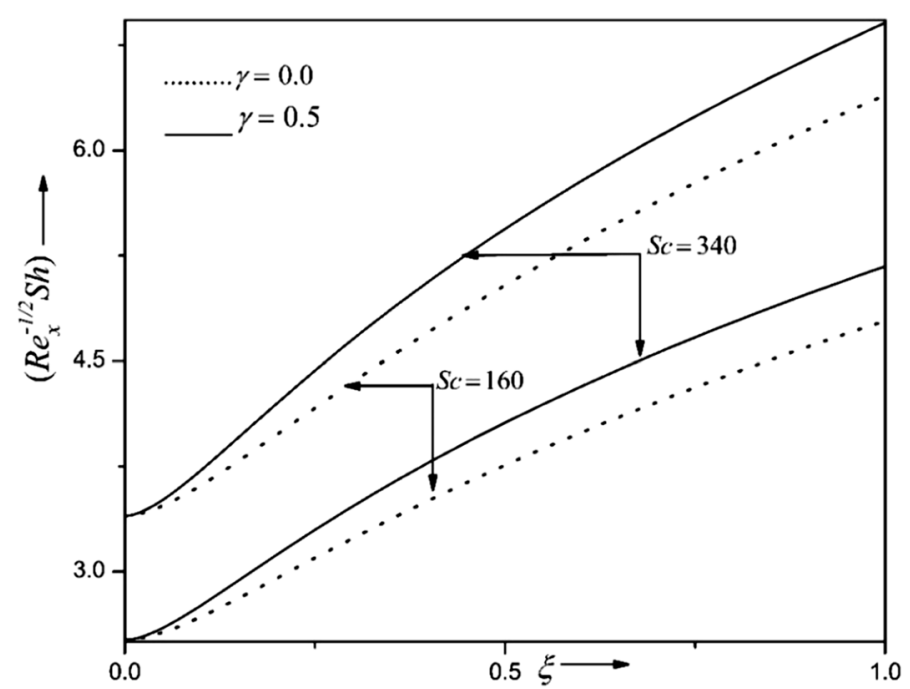

Fig. 9 Effect of $\gamma$ and $S c$ on $\left(\operatorname{Re}_{x}^{-1 / 2} \mathrm{Sh}\right)$ when $\mathrm{Nb}=0.1, \mathrm{Ec}=0.1, \mathrm{Ri}=10, \mathrm{Nc}=0.1, \operatorname{Pr}=7, \operatorname{Re}=10, m=0.0909$, $M=0.1, \xi=0.5$, Le $=10, \mathrm{Nt}=0.1, \beta_{c}=0.1$ and $\mathrm{Nr}=0.1$

Schmidt number $S c$. The species concentration characteristics are found to decrease for higher $\gamma$ and Sc values, the mass transport rate increases, significantly. Particularly, for rising $\gamma$, the temperature variance between the wedge and the surrounding nanofluid increases. These factors decline the magnitude of non-dimensional concentration distribution and, consequently, increase the mass transport rate. In addition to this, the nondimensional concentration distribution is greater for liquid hydrogen $(\mathrm{Sc}=160)$ than liquid oxygen $(\mathrm{Sc}=340)$. For larger $\mathrm{Sc}$ values, the mass diffusivity is found to be lower. This causes a declination in thickness of the concentration boundary layer while dramatically rises the mass transport rate.

Figures 10 and 11 are plotted for variations in volume fraction of nanoparticle distribution $S(\xi, \eta)$ and nanoparticle Sherwood number $\left(\operatorname{Re}_{x}^{-1 / 2} \mathrm{NSh}\right)$ for varying Lewis number Le and nanoparticle buoyancy ratio parameter $\mathrm{Nr}$. The magnitude of $S(\xi, \eta)$ is found to decrease for higher Le values, while $\left(\operatorname{Re}_{x}^{-1 / 2} \mathrm{NSh}\right)$ increases. Moreover, the opposite phenomenon is observed for higher Nr values. Physical reason is that the haphazard movement of nanoparticles reduces with increasing Le values, which results in diminishing the $S(\xi, \eta)$. Consequently, the diffusion of nanoparticles near the wedge surface increases and results in the enhancement of magnitude of nanoparticle Sherwood number. Further, the buoyancy due to nanoparticles increases with the enhancing $\mathrm{Nr}$ values, which increases the $S(\xi, \eta)$, while it decreases the magnitude of $\left(\operatorname{Re}_{x}^{-1 / 2} \mathrm{NSh}\right)$. For example, at $\xi=1$, as $\mathrm{Nr}$ rising from 0 to 1 , mass transfer rate of nanoparticles upsurges about $15 \%$ and $8 \%$ for $\mathrm{Le}=5$ and $\mathrm{Le}=10$, respectively.

\section{Influence of applied magnetic field parameter}

Effects of thermophoresis parameter Nt and magnetic field parameter $M$ on temperature profile $G(\xi, \eta)$ are exhibited in Fig. 12. The higher Nt and $M$ values increase the nanofluid velocity. In particular, the physical parameter $\mathrm{Nt}$ is driven by the temperature gradient. As a result, nanoparticles move from hotter side of wedge to the colder region 


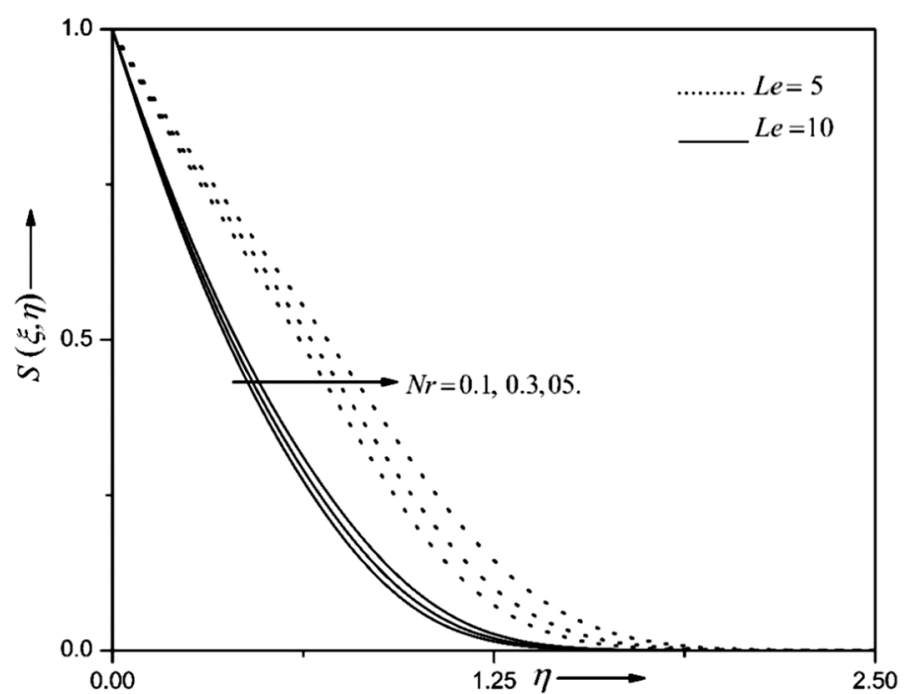

Fig. 10 Influence of $\mathrm{Nr}$ and Le on $S(\xi, \eta)$ for $m=0.0909, \operatorname{Re}=10, \xi=0.5, M=0.1, \mathrm{EC}=0.1, \mathrm{Ri}=10, \mathrm{Nc}=0.1$, $\mathrm{Nb}=0.1, \gamma=0.1, \mathrm{Nt}=0.1, \mathrm{Sc}=160, \operatorname{Pr}=7$ and $\beta_{c}=0.1$

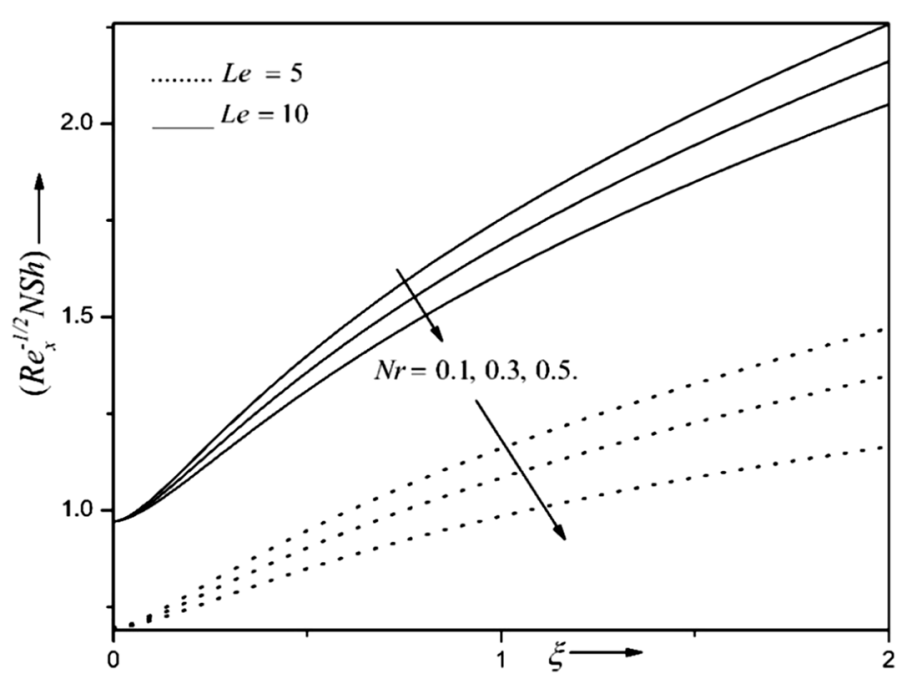

Fig. 11 Effect of $\mathrm{Nr}$ and Le on $\left(\operatorname{Re}_{x}^{-1 / 2} \mathrm{NSh}\right)$ when $\mathrm{EC}=0.1, \mathrm{Ri}=10, \mathrm{Nc}=0.1, \gamma=0.1, \mathrm{Nt}=0.1, \mathrm{Sc}=160$, $\mathrm{Nb}=0.1, m=0.0909, \operatorname{Re}=10, M=0.1, \operatorname{Pr}=7$ and $\beta_{C}=0.1$

of fluid. In addition to this, the presence of magnetic parameter $M$ eventually leads to a drag force near the wedge surface, i.e., in presence of $M$, the Lorentz force suppresses the fluid particle motion and results in enhancement of the temperature of the nanoliquid, significantly.

\section{Numerical validation}

In this work, we have compared the surface friction coefficient $\left(\operatorname{Re}_{x}^{1 / 2} C_{f}\right)$ and energy transfer rate $\left(\operatorname{Re}_{x}^{-1 / 2} \mathrm{Nu}\right)$ with the findings of Kumari et al. [16] and Singh et al. [18] in Table 1 for different wedge angle parameter $(m)$ values when $\xi=\mathrm{Ri}=\mathrm{Re}=\mathrm{Nb}=\mathrm{Ec}=$ $\mathrm{Nr}=\mathrm{M}=\mathrm{Le}=\mathrm{Nt}=\mathrm{Nc}=\beta_{c}=\gamma=0$. The comparison results of gradients values in the 


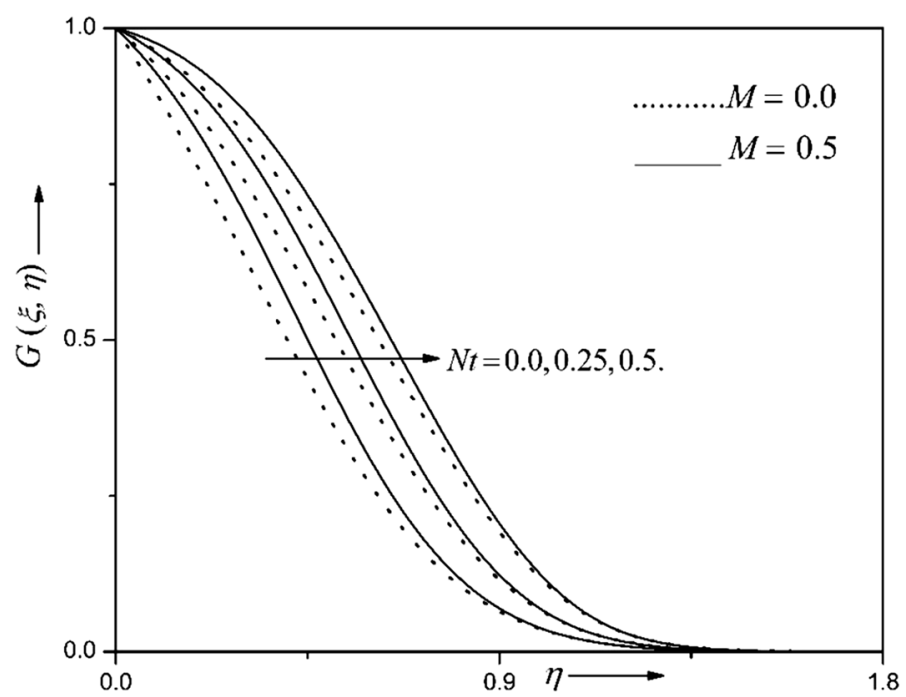

Fig. 12 Influence of $M$ and $\mathrm{Nt}$ on $G(\xi, \eta)$ when $\gamma=0.1, \mathrm{Nc}=0.1, \mathrm{Nb}=0.1, m=0.0909, \mathrm{Ri}=10, \mathrm{Ec}=0.1$, $\operatorname{Re}=10, \xi=0.5$, Le $=10, \mathrm{Sc}=160, \operatorname{Pr}=7, \beta_{c}=0.1$ and $\mathrm{Nr}=0.1$

Table 1 A comparison of drag coefficient and Nusselt number values obtained in the current work through the results of Kumari et al. [16] and Singh et al. [18] for different wedge angles

\begin{tabular}{|c|c|c|c|c|c|c|}
\hline \multirow{2}{*}{$\begin{array}{l}\text { wedge angle } \\
\text { parameter }(m)\end{array}$} & \multicolumn{2}{|c|}{ Kumari et al. [16] } & \multicolumn{2}{|c|}{ Singh et al. [18] } & \multicolumn{2}{|c|}{ Present results } \\
\hline & $F_{\eta}(\xi, 0)$ & $G_{\eta}(\xi, 0)$ & $F_{\eta}(\xi, 0)$ & $G_{\eta}(\xi, 0)$ & $F_{\eta}(\xi, 0)$ & $G_{\eta}(\xi, 0)$ \\
\hline 0 & 0.46975 & 0.42079 & 0.46975 & 0.42046 & 0.46975 & 0.42076 \\
\hline 0.0141 & 0.50472 & 0.42635 & 0.50481 & 0.42606 & 0.50480 & 0.42610 \\
\hline 0.0435 & 0.56904 & 0.43597 & 0.56895 & 0.43559 & 0.56899 & 0.43559 \\
\hline 0.0909 & 0.65501 & 0.44770 & 0.65490 & 0.44742 & 0.65499 & 0.44742 \\
\hline 0.1429 & 0.73202 & 0.45728 & 0.73196 & 0.45705 & 0.73198 & 0.45706 \\
\hline 0.2000 & 0.80214 & 0.46534 & 0.80208 & 0.46511 & 0.80210 & 0.46515 \\
\hline 0.3333 & 0.92766 & 0.47840 & 0.92767 & 0.47815 & 0.92767 & 0.47820 \\
\hline
\end{tabular}

Table 2 The values obtained to the gradients for without nonlinear mixed convection $(\gamma=0)$ and in non-occurrence, as well as occurrence of applied magnetic field parameter values

\begin{tabular}{llr}
\hline Gradients & \multicolumn{2}{l}{ Without nonlinear mixed convection $(\gamma=0)$} \\
\cline { 2 - 3 } & $M=\mathbf{0}$ & $M=\mathbf{1}$ \\
\hline$\left|\mathrm{Re}^{-1 / 2} C_{f}\right|$ & 17.6157 & 19.4903 \\
$\left|\mathrm{Re}^{-1 / 2} \mathrm{Nu}\right|$ & 0.7464 & 0.3141 \\
$\left|\mathrm{Re}^{-1 / 2} \mathrm{Sh}\right|$ & 4.7435 & 4.9362 \\
$\left|\mathrm{Re}^{-1 / 2} \mathrm{NSh}\right|$ & 16.9972 & 18.9521 \\
\hline
\end{tabular}

present study are in good consistency with the previously established results. Tables 2 and 3 present the impacts of the coefficient of drag, energy transport rate, Sherwood number, nanoparticle Sherwood number for without and with of quadratic mixed convection parameter. Also, the surface gradients are obtained in these tables for the absence and presence of applied magnetic field, i.e., $M=0$ and $M=1$. for $m=0.0909$, 
Table 3 The values obtained for the gradients with nonlinear mixed convection $(\gamma=1)$ and in nonoccurrence, as well as occurrence of applied magnetic field for the parameter values

\begin{tabular}{|c|c|c|}
\hline \multirow[t]{2}{*}{ Gradients } & \multicolumn{2}{|c|}{ With nonlinear mixed convection $(\gamma=1)$} \\
\hline & $M=0$ & $M=1$ \\
\hline$\left|\operatorname{Re}^{-1 / 2} C_{f}\right|$ & 23.9029 & 26.3190 \\
\hline$\left|\mathrm{Re}^{-1 / 2} \mathrm{Nu}\right|$ & 0.8403 & 0.4655 \\
\hline$\left|\mathrm{Re}^{-1 / 2} \mathrm{Sh}\right|$ & 5.4626 & 5.4764 \\
\hline$\left|\mathrm{Re}^{-1 / 2} \mathrm{NSh}\right|$ & 22.3629 & 23.4753 \\
\hline
\end{tabular}

$\mathrm{Nt}=0.1, \mathrm{Nc}=0.1, \mathrm{Nb}=0.1, \mathrm{Ec}=0.1, \mathrm{Nr}=0.1, \mathrm{Le}=10, \mathrm{Ri}=10, \mathrm{Re}=10$, and $\beta_{c}=0.1$. It is perceived from Tables 2 and 3, the magnitudes of surface gradients rise significantly for $\gamma=1$, irrespective of magnetic parameter values. On the other hand, the magnitudes of skin friction coefficient, mass transfer rate, nanoparticle mass transfer rate increase, while the heat transfer rate reduces considerably for enhancing magnetic parameter, irrespective of nonlinear mixed convection parameter values.

\section{Conclusions}

The numerical investigation has been carried out for MHD double diffusive nonlinear mixed convective nanofluid flow over a vertical wedge with liquid hydrogen diffusion. The following major cases have been studied in this problem. Analysis of double diffusive nonlinear mixed convection with nanoparticles. Brownian motion and thermophoresis effects are analyzed. Influence of applied magnetic field over a wedge. Impacts of diffusion of liquid hydrogen is analyzed. Also, the comparison results of gradients values in the present study are in good agreement with the previously published results. The innovative findings of the present analysis are summarized as follows:

- For different values of wedge angle parameter, the fluid particles undergo friction with wedge surface and it enhances the magnitude of drag coefficient.

- As quadratic combined convection parameter rises, the temperature gap between the surface and the fluid expands. This lowers the temperature of the nanofluid thereby enhancing the heat transfer rate strength, significantly.

- The temperature gradient in the thermophoresis effect results in rising the nanoliquid's temperature.

- The dispersed nanoparticles generate the friction near the wall of wedge and delay the transfer of heat from the hot wedge to surrounding ambient liquid.

- The nanoparticles in the base fluid enhance the friction at the vicinity of the wall and reduce the rate of heat transfer from hot surface of wedge to the cold ambient fluid.

- In occurrence of magnetic field, the Lorentz force suppresses the nanoliquid motion, which causes an augmentation of the nanofluid's temperature.

- Higher Schmidt number values result in lower mass diffusivity which decreases the concentration profile and increases the mass transportation rate.

- Increase in Lewis number reduces the magnitude nanoparticle volume fraction profile and nanoparticle mass transport rate increases. 


\section{Abbreviations}

FDM: Finite difference method; MHD: Magnetohydrodynamics; NDT: Nonlinear changes in density temperature; NDTC: Nonlinear changes in density temperature and species concentration; NPDEs: Nonlinear partial differential equations.

\section{List of symbols}

$\operatorname{Pr}=\frac{v}{\alpha_{m}}:$ Prandtl number; Le $=\frac{v}{D_{B}}:$ Lewis number; $\mathrm{Sc}=\frac{v}{D_{s}}:$ Schmidt number for liquid hydrogen; $\mathrm{Ri}=\frac{\mathrm{Gr}}{\operatorname{Re}^{2}}$ : Richardson number; Ec $=\frac{U_{\infty}^{2}}{C_{n f}\left(T_{w}-T_{\infty}\right)}$ : Eckert number; Gr $=\frac{g \beta_{1}\left(1-\phi_{\infty}\right)\left(T_{w}-T_{\infty}\right) L^{3}}{\beta_{3}\left(C_{w} \nu^{2} C_{\infty}\right)} \cos \left(\frac{\pi \alpha}{2}\right)$ : Grashof number; $\mathrm{Nb}=\mathrm{JD}_{B} \frac{\left(\phi_{w}-\phi_{\infty}^{n f}\right)}{v}$ : Brownian diffusion parameter; $\mathrm{Nc}=\frac{\beta_{3}\left(C_{w}{ }^{2}-C_{\infty}\right)}{\beta_{1}\left(T_{w}-T_{\infty}\right)}$ : Buoyancy ratio parameter; $\mathrm{Nt}=\mathrm{JD}_{T} \frac{\left(T_{w}-T_{\infty}\right)}{\nu T_{\infty}}$ :Thermophoresis parameter $J=\frac{\rho_{p} C_{p p}}{\rho C_{n f}}$ : Ratio of heat capacity of nanoparticles to the heat capacity of the nanofluid; $\mathrm{Nr}=\left(\frac{\rho_{p}-\rho}{\rho}\right) \frac{\left(\phi_{w_{0}}-\phi_{\infty}\right)}{\beta_{1}\left(T_{w}-T_{\infty}\right)\left(1-\phi_{\infty}\right)} \cos \left(\frac{\pi \alpha}{2}\right)$ : Nanoparticle buoyancy ratio parameter; $\gamma=\frac{\beta_{2}}{\beta_{1}}\left(T_{w}-T_{\infty}\right)$ : Nonlinear mixed convection parameter; $M=\frac{B_{0}}{U_{e}} \sqrt{\frac{\sigma v}{\rho}}$ : Magnetic parameter; $\operatorname{Re}=\frac{U_{\infty} L}{}, \operatorname{Re}_{x}=\frac{U_{e} x}{v}$ : Reynolds number defined in terms of $L$ and $x$, respectively; $C_{n f}$ : Heat capacity of nanofluid $\left(\mathrm{JK}^{-1}\right) ; D_{B}$ : Brownian diffusion coefficient $\left(\mathrm{m}^{2} \mathrm{~s}^{-1}\right) ; D_{T}$ : Thermophoretic coefficient $\left(\mathrm{m}^{2} \mathrm{~s}^{-1}\right) ; D_{s}$ : Mass diffusion of liquid hydrogen; $F$ : Non-dimensional velocity profile; $G$ : Non-dimensional temperature profile; $H$ : Non-dimensional concentration profile; $S$ : Non-dimensional nanoparticle volume fraction profile; $T_{w}, T_{\infty}$ : Temperature at surface and ambient conditions $(K) ; C_{w}, C_{\infty}$ : Concentration of liquid hydrogen at the surface and away from the surface; $x, y$ : Distances along $x$ and $\boldsymbol{y}$ co-ordinates $(\mathrm{m}) ; \overline{\boldsymbol{x}}$ : Dimensionless axial distance; $\boldsymbol{u}, \boldsymbol{v}$ : The velocity component in $\boldsymbol{x}$ and $\boldsymbol{y}$ directions; $U_{e}$ : Free stream velocity at the edge of the boundary layer $\left(\mathrm{ms}^{-1}\right) ; U_{\infty}$ : Constant free stream velocity $\left(\mathrm{ms}^{-1}\right)$; $L$ : Characteristic length $(m) ; \Omega$ : Total angel of wedge (radians); $m$ : Wedge angle parameter (radians); $B_{0}$ : Constant magnetic field strength, $C_{p p}$ : Specific heat capacity of nanoparticles $\left(\mathrm{JK}^{-1}\right) ; \alpha$ : Hartree pressure gradient; $\sigma$ : Electrical conductivity of the fluid $(\mathrm{S} / \mathrm{m}) ; \beta_{1} \beta_{2}$ : Linear and nonlinear thermal expansion coefficients $\left(\mathrm{K}^{-1}\right) ; g$ : Acceleration due to gravity $\left(\mathrm{m} \mathrm{s}^{-2}\right) ; \rho$ : Density of nanofluid $\left(\mathrm{kg} \mathrm{m}^{-3}\right) ; \phi_{w}, \phi_{\infty}$ : Nanoparticle volume fraction at the wall and ambient conditions; $\alpha_{m}$ : Thermal diffusivity of nanofluid; $\beta_{3} \beta_{4}$ : Linear and nonlinear concentration expansion coefficients; $\rho_{p}$ : Nanoparticle mass density $\left(\mathrm{kg} \mathrm{m}^{-3}\right) ; \beta_{c}$ : Quadratic combined convection parameter for liquid hydrogen concentration; $\gamma$ : Quadratic combined convection parameter for temperature.

\section{Acknowledgements}

None.

\section{Authors' contributions}

PMP, MK contributed to conception, investigation and methodology; MK contributed to software; PMP, MK contributed to writing — original draft; all authors have read and approved the manuscript.

\section{Funding}

Funding information is not applicable/no funding was received.

\section{Availability of data and materials}

Not applicable.

\section{Declarations}

\section{Competing interests}

The authors declare that they have no known competing interests.

\section{Author details}

'Department of Mathematics, Karnatak University, Pavate Nagar, Dharwad 580003, India. ${ }^{2}$ Wrangler Dr. D. C. Pavate Institute of Mathematical Sciences (PIMSci.), Karnatak University, Pavate Nagar, Dharwad 580003, India.

Received: 27 May 2021 Accepted: 29 November 2021

Published online: 14 December 2021

\section{References}

1. Abdelsalam, S.I., Zaher, A.Z.: Leveraging elasticity to uncover the role of Rabinowitsch suspension through a wavelike conduit: consolidated blood suspension application. Mathematics 2021, 9 (2008)

2. Shankar, B.M., Kumar, J., Shivakumara, I.S.: Magnetohydrodynamic instability of mixed convection in a differentially heated vertical channel. Eur. Phys. J. Plus 134, 53 (2019)

3. Shankar, B.M., Kumar, J., Shivakumara, I.S.: Stability of mixed convection in a differentially heated vertical fluid layer with internal heat sources. Fluid Dyn. Res. (2019). https://doi.org/10.1088/1873-7005/ab2d50

4. Abumandour, R.M., Eldesoky, I.M., Kamel, M.H., Ahmed, M.M., Abdelsalam, S.I.: Peristaltic thrusting of a thermalviscosity nanofluid through a resilient vertical pipe. Z. Naturforschung A 75, 727-738 (2020)

5. Shankar, B.M., Kumar, J., Shivakumara, I.S.: Numerical investigation of the stability of mixed convection in a differentially heated vertical porous slab. Appl. Math. Comput. 389, 125486 (2021)

6. Sinha, P.C.: Fully developed laminar free convection flow between vertical parallel plates. Chem. Eng. Sci. 24, 33-38 (1969) 
7. Vajravelu, K., Sastri, K.S.: Fully developed laminar free convection flow between two parallel vertical walls. Int. J. Heat Mass Transf. 20, 655-660 (1977)

8. Kameswaran, P.K., Sibanda, P., Partha, M.K., Murthy, P.V.S.N.: Thermophoretic and nonlinear convection in nonDarcy porous medium. J. Heat Transf. 136, 042601-042609 (2014)

9. Shaw, S., Kameswaran, P.K., Sibanda, P.: Effects of slip on nonlinear convection in nanofluid flow on stretching surfaces. Bound. Value Probl. (2016). https://doi.org/10.1186/s13661-015-0506-2

10. Hayat, T., Qayyum, S., Shehzad, S.A., Alsaedi, A.: Magnetohydrodynamic three-dimensional nonlinear convection flow of Oldroyd-B nanoliquid with heat generation/absorption. J. Mol. Liq. 230, 641-651 (2017)

11. Khan, M.I., Khan, T.A., Qayyum, S., Hayat, T., Khan, M.I., Alsaedi, A.: Entropy generation optimization and activation energy in nonlinear mixed convection flow of a tangent hyperbolic nanofluid. Eur. Phys. J. Plus 133, 329 (2018). https://doi.org/10.1140/epjp/i2018-12093-y

12. Hayat, T., Rashid, M., Alsaedi, A., Asghar, S.: Nonlinear convective flow of Maxwell nanofluid past a stretching cylinder with thermal radiation and chemical reaction. J. Braz. Soc. Mech. Sci. Eng. 41, 86 (2019). https://doi.org/ 10.1007/s40430-019-1576-3

13. Uddin, I., Akhtar, R., Khan, A.R., Zhiyu, Z., Islam, S., Shoaib, M., Raja, M.A.Z.: Numerical treatment for fluidic system of activation energy with non-linear mixed convective and radiative flow of magneto nanomaterials with Navier's velocity slip. AIP Adv. 9, $055210-055211$ (2019)

14. Falkner, V.M., Khan, S.W.: Some approximate solutions of boundary layer equations. Philos. Mag. Ser. 7, 865-896 (1931)

15. Kafoussias, N.G., Nanousis, N.D.: Magnetohydrodynamic laminar boundary layer flow over vertical wedge with suction or injection. Can. J. Phys. 75, 733-745 (1997)

16. Kumari, M., Takhar, H.S., Nath, G.: Mixed convection flow over a vertical wedge embedded in a highly porous medium. Heat Mass Transf. 37, 139-146 (2001)

17. Kandasamy, R., Muhaimin, I., Hashim, I., Kasmani, R.M.: Thermophoresis and chemical reaction effects on nonDarcy mixed convective heat and mass transfer past a porous wedge with variable viscosity in presence of suction or injection. Nucl. Eng. Des. 238, 2699-2705 (2008)

18. Singh, P.J., Roy, S., Ravindran, R.: Unsteady mixed convection flow over a vertical wedge. Int. J. Heat Mass Transf. 52, 415-421 (2009)

19. Uddin, Z., Kumar, M.: Hall and ion-slip effect on MHD boundary layer flow of micro polar fluid past a wedge. Sci. Iran. B. 20, 467-476 (2013)

20. Deka, R.K., Sharma, S.: Magnetohydrodynamic mixed convection flow past as wedge under variable temperature and chemical reaction. Am. J. Comput. Appl. Math. 3, 74-80 (2013)

21. Ganapathirao, M., Ravindran, R., Momoniat, E.: Effects of chemical reaction, heat and mass transfer on unsteady mixed convection boundary layer flow a wedge with heat generation/absorption in presence of suction/injection. Heat Mass Transf. 51, 289-300 (2015)

22. Choi, S.U.S., Eastman, J.A.: Enhancing thermal conductivity of fluids with nanoparticles. In: ASME International Mechanical Engineering Congress Exposition, pp. 99-105. San Francisco CA (1995)

23. Gorla, R.S.R., Chamka, A.J., Rashad, A.M.: Mixed convective boundary layer flow over a vertical wedge embedded in a porous medium saturated with a nanofluid: natural convection dominated regime. Nanoscale Res. Lett. $\mathbf{6}$, 207 (2011). https://doi.org/10.1186/1556-276X-6-207

24. Kahalerras, H., Fersadou, B., Nessab, W.: Mixed convection heat transfer and entropy generation analysis of copper-water nanofluid in a vertical channel with non-uniform heating. SN Appl. Sci. 2, 76 (2019). https://doi.org/ 10.1007/s42452-019-1869-2

25. Singh, R., Bishnoi, J., Tyagi, V.K.: Triple diffusive convection with Soret-Dufour effects in a Maxwell nanofluid saturated in a Darcy porous medium. SN Appl. Sci. 2, 704 (2020). https://doi.org/10.1007/s42452-020-2462-4

26. Rahman, M.M., Al-Lawatia, M.A., Eltayeb, I.A., Al-Salti, N.: Hydrodynamic slip flow of water based nanofluid past a wedge with convective surface in the presence of heat generation or absorption. Int. J. Therm. Sci. 57, 172-182 (2012)

27. Khan, M.S., Karim, I., Islam, M.S., Wahiduzzaman, M.: MHD Boundary layer radiative, heat generating and chemical reacting flow past a wedge moving in a nanofluid. Nano Converg 1, 20 (2014). https://doi.org/10.1186/ s40580-014-0020-8

28. Kasmani, R.M., Sivasankaran, S., Bhuvaneswari, M., Hussein, A.K.: Analytical and numerical study on convection of nanofluid past a moving wedge with Soret and Dufour effects. Int. J. Numer. Methods Heat Fluid Flow (2016). https://doi.org/10.1108/HFF-07-2016-0277

29. Kasmani, R.M., Sivasankaran, S., Bhuvaneswari, M., Siri, Z.: Effect of chemical reaction on convective heat transfer of boundary layer flow in nanofluid over a wedge with heat generation/absorption and suction. J. Appl. Fluid Mech. 9, 379-388 (2016)

30. Kasmani, R.M., Sivasankaran, S., Bhuvaneswari, M., Alshomrani, A.S.: Soret and Dufour effects on doubly diffusive convection of nanofluid over wedge in the presence of thermal radiation and suction. Sci. Iran. B 26, 2817-2826 (2019)

31. Naveen Kumar, S.B., Shivakumara, I.S., Shankar, B.M.: Exploration of Coriolis force on the linear stability of couple stress fluid flow induced by double diffusive convection. ASME J. Heat Transf. 141, 122502 (2019)

32. Kumar, S.N., Shivakumara, I.S., Shankar, B.M.: Linear and weakly nonlinear double-diffusive magnetoconvection in a non-Newtonian fluid layer. Microgravity Sci. Technol. 32, 629-646 (2020)

33. Elmaboud, Y.A., Abdelsalam, S.I.: DC/AC magnetohydrodynamic-micropump of a generalized Burger's fluid in an annulus. Phys. Scr. 94, 115209 (2019)

34. Bhatti, M.M., Abdelsalam, S.I.:Thermodynamic entropy of a magnetized Ree-Eyring particle-fluid motion with irreversibility process: a mathematical paradigm. J. Appl. Math. Mech. 101, 202000186 (2021)

35. Bhatti, M.M., Alamri, S.Z., Ellahi, R.A.: Sl: intra-uterine particle-fluid motion through a compliant asymmetric tapered channel with heat transfer. J. Therm. Anal. Calorim. 144, 2259-2267 (2021) 
36. Abdelsalam, S.I., Velasco-Hernández, J.X., Zaher, A.Z.: Electro-magnetically modulated self-propulsion of swimming sperms via cervical canal. Biomech. Model Mechanobiol. 20, 861-878 (2021)

37. Rashidi, M.M., Kavyani, N., Abelman, S., Uddin, M.J., Freidoonimehr, N.: Double diffusive magnetohydrodynamic (MHD) Mixed convective slip flow along a radiating moving vertical flat plate with convective boundary condition. PLoS ONE 9, e109404 (2014). https://doi.org/10.1371/journal.pone.0109404

38. Patil, P.M., Kumbarwadi, N., Shashikant, A.: Effects of MHD mixed convection with non-uniform heat source/sink and cross-diffusion over exponentially stretching sheet. Int. J. Numer. Methods Heat Fluid Flow 28, 1256-1278 (2017)

39. Prasad, D.V.K., Chaitanya, G.S.K., Raju, R.S.: Double diffusive effects on mixed convection Casson fluid flow past a wavy inclined plate in presence of Darcian porous medium. Res. Eng. 3, 100019 (2019)

40. Patil, P.M., Doddagoudar, S.H., Hiremath, P.S., Momoniat, E.: Influence of applied magnetic field on mixed convective nanofluid flow past an exponentially stretching surface with roughness. J. Braz. Soc. Mech. Sci. Eng. 41, 562 (2019). https://doi.org/10.1007/s40430-019-2065-4

41. Qiang, X., Siddique, I., Sadiq, K., Shah, N.A.: Double diffusive MHD convective flows of a viscous fluid under influence of the inclined magnetic field, source/sink and chemical reaction. Alex. Eng. J. (2020). https://doi.org/10.1016/j.aej. 2020.07.023

42. Mahajan, A., Sharma, M.K.: Double-diffusive convection in a magnetic nanofluid layer with cross diffusion effects. J. Eng. Math. 115, 67-87 (2019). https://doi.org/10.1007/s10665-019-09992-8

43. Patil, P.M., Roy, S., Pop, I.: Flow and heat transfer over a moving vertical plate in a parallel free stream: role of internal heat generation or absorption. Chem. Eng. Commun. 199, 658-672 (2012)

44. Patil, P.M., Kulkarni, M.: Nonlinear Mixed convective nanofluid flow along moving vertical rough plate. Rev. Mex. Fis. 66, 153-161 (2020)

45. Schlichting, H., Gersten, K.: Boundary Layer Theory. Springer, New York (2000)

46. Buongiorno, J.: Convective transport in nanofluids. ASME J. Heat Transf. 128, 140-250 (2006)

47. Varga, R.S.: Matrix iterative analysis. Prentice-Hall, Englewood Cliffs (2000)

\section{Publisher's Note}

Springer Nature remains neutral with regard to jurisdictional claims in published maps and institutional affiliations.

\section{Submit your manuscript to a SpringerOpen ${ }^{\odot}$ journal and benefit from:}

- Convenient online submission

- Rigorous peer review

- Open access: articles freely available online

- High visibility within the field

- Retaining the copyright to your article

Submit your next manuscript at $\gg$ springeropen.com 